\title{
Effets de l'apport de bois raméal sur la plante et le sol : une revue des résultats expérimentaux
}

\author{
Bernard G. Barthès ${ }^{1}$ \\ Raphaël J. Manlay ${ }^{1,2}$ \\ Olivier Porte ${ }^{1}$ \\ ${ }^{1}$ IRD \\ UMR 210 Eco\&Sols \\ Montpellier SupAgro \\ Bâtiment 12 \\ 2, place Viala \\ 34060 Montpellier cedex 2 \\ France \\ <bernard.barthes@ird.fr> \\ $<$ raphael.manlay@ird.fr> \\ <olivier.porte@yahoo.fr> \\ ${ }^{2}$ AgroParisTech-Engref
}

GFR « Gestion environnementale

des écosystèmes et forêts tropicales "

648, rue Jean-Francois Breton

BP 7355

34086 Montpellier cedex 4

France

<raphael.manlay@agroparistech.fr>

\begin{abstract}
Résumé
L'amendement du sol avec des branches, notamment avec des bois raméaux fragmentés (BRF), suscite un intérêt croissant chez les agriculteurs et les services de vulgarisation, mais la validation scientifique de cette pratique est incomplète. Cet article synthétise les résultats statistiquement significatifs concernant l'effet d'apports enfouis ou paillés (mulch) de BRF sur les cultures et le sol, en milieu tempéré et tropical. L'apport de BRF a généralement un effet positif sur le rendement agricole, sauf pour la culture qui suit immédiatement un premier enfouissement en sol sableux (lequel a surtout été testé en milieu tempéré ; le résultat reste peu documenté en milieu tropical); cet effet négatif peut cependant être limité par un apport d'engrais azoté. Par ailleurs, l'apport de BRF, surtout en mulch, améliore les propriétés physico-hydriques du sol (humidité, porosité, structure), enrichit le sol en matière organique, stimule l'activité biologique, et accroît la disponibilité des nutriments à moyen terme. Les effets des BRF sont modulés par plusieurs facteurs, comme l'essence forestière utilisée et les modalités d'apport (dose, périodicité, dimension des fragments, etc.) ; mais les résultats répertoriés ne permettent guère de formuler des recommandations précises. Par ailleurs, l'intérêt des BRF par rapport aux amendements organiques non ligneux est mal documenté.
\end{abstract}

Mots clés : agriculture alternative ; agroforesterie ; amendement organique ; branche ; sol.

Thèmes : forêts ; productions végétales ; sols.

\section{Abstract \\ Effects of ramial wood amendments on crops and soil: A synthesis of experimental results}

Increasing attention is being paid by farmers and extension services to soil amendment with small branches, especially as chipped ramial wood (CRW), but the scientific validation of this practice is incomplete. The present work summarizes statistically significant results regarding the effects of such branch amendments, both buried and mulched, on crop yield and soil properties in temperate and tropical regions. Broadly speaking, soil amendment with CRW has a positive effect on crop yield, except for the crop that immediately follows the first burying of CRW in sandy soils (which has mainly been tested in temperate regions); however this negative effect can be limited when nitrogen is simultaneously applied. Moreover, CRW application increases soil organic matter content, stimulates soil biological activities, improves medium-term nutrient availability and - especially as mulch - soil hydro-physical properties (moisture, porosity, structure, etc.). The effects of CRW application can be affected by several factors such as tree species and amendment characteristics (amount, periodicity, chip size, etc.), however the results available do not allow precise recommendations. Moreover, the benefit of CRW as compared with non-woody amendments is poorly documented.

Key words: agroforestry; alternative agriculture; branches; organic amendments; soil.

Subjects: forestry; soils; vegetal productions.

Pour citer cet article: Barthès BG, Manlay RJ, Porte O. Effets de l'apport de bois raméal sur la plante et le sol: une revue des résultats expérimentaux. Cah Agric 2010; 19: 280-7. DOI: 10.1684/agr.2010.0412. 
E cosystèmes naturels et agrosystèmes diffèrent par le degré de fermeture des cycles biogéochimiques, du fait de leurs complexités structurale et fonctionnelle contrastées (Odum, 1969). La résilience des premiers est assurée par des mécanismes de maintenance biologique. En revanche les seconds sont très ouverts; leur viabilité dépend du mode de gestion du sol et notamment de la compensation des pertes en minéraux et carbone (C). La gestion conventionnelle des agrosystèmes favorise une maintenance de substitution, visant à conserver certaines propriétés du sol dans des valeurs acceptables (Izac et Swift, 1994). Apports d'intrants minéraux et travail du sol permettent ainsi de compenser les pertes en éléments et de maintenir les propriétés physiques du sol, mais ils peuvent avoir des conséquences agronomiques et environnementales négatives (Tilman et al., 2002).

Face à cette stratégie, l'apport d'amendements organiques est perçu comme une alternative durable de gestion des sols (Manlay et al., 2007). Cependant, cette pratique est souvent limitée par la disponibilité de la ressource et la maîtrise délicate des dynamiques de décomposition des amendements (Kumar et Goh, 2000). L'émergence scientifique de l'agroécologie (Altieri, 2002) répond entre autres à la nécessité de lever ces limitations. L'agroécologie repose sur l'hypothèse que la pérennité des écosystèmes naturels dans un contexte local dépend de traits structuraux et fonctionnels propres, et propose leur transfert aux agrosystèmes via des stratégies d'imitation (Ewel, 1999). L'amendement du sol avec des branches d'arbres, ou bois raméaux (BR), s'inscrit ainsi dans une démarche d'imitation des écosystèmes arborés. Cette technique, inaugurée au Canada il y a une vingtaine d'années, consiste à enfouir dans le sol superficiel des rameaux de faible diamètre (Lemieux, 1996). Mises au point en climat tempéré, les recommandations portent en particulier sur l'utilisation de rameaux de diamètre inférieur à $7 \mathrm{~cm}$ et leur broyage, d'où le terme de bois raméaux fragmentés (BRF) pour désigner le matériau et la technique. Celle-ci vise à rapprocher le statut biologique du sol cultivé de celui du sol forestier supposé d'origine.

L'objectif du présent article est de synthétiser les résultats expérimentaux concernant les effets d'apports de BR sur la plante et le sol, en s'appuyant sur ceux ayant donné lieu à traitement statistique.
Ces travaux font majoritairement référence à l'application de BRF, mais des pratiques voisines sont également considérées, comme le mulch de branches peu fragmentées. Cette synthèse est présentée par type de variables, concernant la plante, puis le sol : propriétés physiques et hydriques, chimiques et d'échange, matière organique, biologie. Elle distingue l'enfouissement de BRF en zone tempérée, en zone tropicale, puis les mulchs ligneux en zone tropicale (deux références seulement évoquant des mulchs de branches, plusieurs travaux sur les mulchs de copeaux de bois sont aussi considérés). Les résultats dits significatifs le sont au seuil de $5 \%$. L'ensemble des résultats est condensé dans le tableau 1.

\section{Effets sur la plante cultivée}

\section{Enfouissement en zone tempérée (Canada)}

Sur sol sablo-graveleux fertilisé en phosphore (P) et potassium (K), N'dayegamiye et Dubé (1986) apportent 12,5, 25 ou $50 \mathrm{t} /$ ha de matière sèche (MS) de BRF (feuillus) tous les deux ans, avec ou sans lisier. Par rapport à ceux du témoin homologue sans BRF, les rendements avec BRF (avec ou sans lisier) sont inférieurs significativement la première année (blé, - 20 à - $80 \%$ ), supérieurs parfois significativement la deuxième année (prairie, + 20 à $190 \%$ ) puis, après un deuxième apport de BRF, la troisième année (orge, 0 à $+10 \%$ ), et significativement supérieurs la quatrième année (blé, + 30 à 75\%); en moyenne sur 4 ans, ils sont 5 à $70 \%$ supérieurs avec BRF. L'augmentation des rendements est expliquée par celle de la disponibilité en azote $(\mathrm{N})$.

Sur sol sableux fertilisé en PK, Beauchemin et al. (1990) observent un rendement en pomme de terre plus faible les deux années suivant l'apport de 25 tMS/ha de $\mathrm{BRF}$ de feuillus et conifères que sans BRF (-50 puis - $25 \%$ ). L'utilisation de conifères semble en cause. Mais les rendements sont significativement plus élevés avec apport complémentaire de $\mathrm{N}$ que sans BRF. Le compostage des BRF ne modifie pas leur effet.

Sur sol limono-argileux, Larochelle (1994) constate que le rendement en orge dimi- nue de 13 à $34 \%$ l'année de l'apport de BRF (15 ou 30 tMS/ha) mais qu'un complément azoté peut compenser la baisse. Pour une culture fertilisée de pomme de terre sur sol sableux après prairie, Tremblay et Beauchamp (1998) notent que l'apport de BRF n'a pas d'effet sur les pathogènes responsables de la galle commune et de la rhizoctonie.

Beauchemin et al. (1992b) constatent que les extraits hydrosolubles de BRF frais de feuillus et résineux réduisent la germination du cresson ; l'effet est accru lorsque les BRF sont broyés, mais très atténué après compostage.

\section{Enfouissement en zone tropicale}

En Côte d'Ivoire (pluviosité annuelle moyenne - PAM : $1150 \mathrm{~mm}$ ), sur sol argileux après jachère, Aman (1996) étudie l'effet de l'enfouissement de 16 à 26 tMS/ha de BRF de cinq essences, avec ou sans engrais NPK, sur les rendements en maïs de l'année. Par rapport aux témoins sans BRF, les rendements augmentent significativement avec BRF (+ 100 à $200 \%$ ) sauf avec Acacia auriculiformis sans engrais (+ $20 \%)$.

À Saint-Domingue (PAM : 800-1 200 mm), Gómez (2003) observe aussi une augmentation significative du rendement en maïs après enfouissement de 11 tMS/ha de BRF (40-56 \%).

$\mathrm{Au}$ contraire, sur sol sableux après oignons au Sénégal (PAM : 700 mm), Soumare et al. (2002) notent une baisse souvent significative des rendements en tomate (- 2 à - $11 \%)$ et des teneurs en $\mathrm{N}$ et $\mathrm{P}$ des feuilles après apport de 8 à 31 tMS/ha de BRF de Casuarina equisetifolia. Mais les rendements de la seconde culture suivant l'apport sont significativement plus élevés que ceux du témoin (40-60\%). De plus, les rendements augmentent quand l'apport passe de 8 à 16 tMS/ha de BRF, mais les auteurs n'expliquent pas pourquoi ils sont plus faibles avec 31 qu'avec $8 \mathrm{tMS} / \mathrm{ha}$.

Au Kenya (PAM : $1800 \mathrm{~mm}$ ), sur sol argilo-sableux fertilisé en $\mathrm{K}$ après maïsharicot sans aucun apport, Kwabiah et al. (2003) observent durant deux années que l'enfouissement de 5 tMS/ha/an de $\mathrm{BRF}$ conduit en général à des rendements en maïs qui ne diffèrent pas de ceux obtenus avec engrais minéral NP, sauf avec BRF de légumineuses, qui donnent de moins bons résultats. 
Tableau 1. Synthèse des travaux cités.

Table 1. Synthesis of the cited references.

\begin{tabular}{|c|c|c|c|c|c|c|c|c|}
\hline \multirow[t]{2}{*}{ Réf. } & \multirow[t]{2}{*}{ Climat, texture } & \multirow{2}{*}{$\begin{array}{l}\text { Modalité différant } \\
\text { du témoin }\end{array}$} & \multirow{2}{*}{$\begin{array}{l}\text { Apports ligneux } \\
\text { (tMS/ha)* }\end{array}$} & \multicolumn{3}{|c|}{ Rendement $^{1}$} & \multirow[t]{2}{*}{ Physique et chimie du sol } & \multirow{2}{*}{$\begin{array}{l}\text { Matière organique } \\
\text { et biologie du sol }\end{array}$} \\
\hline & & & & Cult.1 & Cult.2 & Cult.4 & & \\
\hline \multirow[t]{2}{*}{1,2} & Tempéré, sableux & BRF enfoui & $12,5,25$ ou $50 / 2$ ans & - & $(+)^{5}$ & + & $\mathrm{CEC}=$ à 4 ans, agrégation $=$ à 9 ans & $\begin{array}{l}\% \mathrm{C}+\text { et }+, \mathrm{N}=\text { et }+ \\
\mathrm{C} / \mathrm{N}+\text { et }=\text { à } 4 \text { et } 9 \text { ans }\end{array}$ \\
\hline & & BRF enfoui avec $\mathrm{N}$ & $12,5,25$ ou $50 / 2$ ans & - & $+{ }^{6}$ & + & $\mathrm{CEC}=$ à 4 ans, agrégation= à 9 ans & $\begin{array}{l}\% \mathrm{C}+, \mathrm{N}=\text { et } \mathrm{C} / \mathrm{N}+ \\
\text { à } 4 \text { et } 9 \text { ans }\end{array}$ \\
\hline \multirow[t]{2}{*}{3,4} & Tempéré, sableux & BRF enfoui & 25 (1 fois) & - & $(-)$ & nd & Minéralisation N - ; humidité an2+ & \\
\hline & & BRF enfoui avec $\mathrm{N}$ & 25 (1 fois) & + & + & nd & Minéralisation N - ; humidité an2+ & \\
\hline \multirow[t]{2}{*}{5} & Tempéré, & BRF enfoui & 15 ou 30 (1 fois) & $(-)$ & nd & nd & \% nitrate - & Mésofaune + ou (+) \\
\hline & limono-argileux & BRF enfoui avec N & 15 (1 fois) & $=$ & nd & nd & & Mésofaune + ou (+) \\
\hline 6 & $\begin{array}{l}\text { Tempéré, } \\
\text { sablo-limoneux }\end{array}$ & BRF enfoui & $150 * *$ (1 fois) & nd & nd & nd & $\begin{array}{l}\text { \% nitrate an } 1-\text {, an2 }=\text {; } \\
\text { Humidité }+ \text {, agrégation an2+ }\end{array}$ & $\begin{array}{l}\% \mathrm{C} \text { et } \mathrm{N} \text { an } 1=\text {, an } 2+; \\
\text { champignons }+ \\
\text { autres microbes an } 1+, \\
\text { an2 }=\end{array}$ \\
\hline 7 & Tempéré, sableux & BRF enfoui & $37.5^{* *}$ /an ou / 2 ans & nd & nd & nd & $\begin{array}{l}\% \mathrm{P}_{\text {ass }} \text { an } 1-, \text { an } 2(-) ; \mathrm{pH}= \\
\text { Humidité an1=, an2+ }\end{array}$ & $\begin{array}{l}\text { Champignons an } 1+\text {, an } 2=\text {; } \\
\text { autres microbes }=; \\
\% \mathrm{C} \text { an } 1=\text {, an } 2+, \mathrm{C} / \mathrm{N}=\end{array}$ \\
\hline \multirow[t]{2}{*}{8} & Tropical subhumide, & BRF enfoui & 16 à 26 (1 fois) & $+{ }^{3}$ & nd & nd & & \\
\hline & argileux & BRF enfoui avec $N$ & 16 à 26 (1 fois) & + & nd & nd & & \\
\hline 9 & $\begin{array}{l}\text { Tropical sec, } \\
\text { sableux }\end{array}$ & BRF enfoui & 8, 16 ou 31 (1 fois) & -4 & + & nd & $\begin{array}{l}\mathrm{CEC}+, \% \mathrm{~K}_{\text {éch }}+, \% \mathrm{P}_{\mathrm{ass}^{-}}, \text {humidité }+ \\
\text { et densité - à la récolte } 2\end{array}$ & $\% \mathrm{C}(+)$ et $\mathrm{N}+$ à la récolte 2 \\
\hline 10 & Tropical subhumide ${ }^{2}$ & BRF enfoui & 11 (1 fois) & + & nd & nd & $\mathrm{pH}=$ et $\mathrm{CEC}(-)$ & $\% \mathrm{C}(+)$ \\
\hline 11 & $\begin{array}{l}\text { Tropical humide, } \\
\text { argilo-sableux }\end{array}$ & $\begin{array}{l}\text { BRF enfoui } \\
\text { sans NP }\end{array}$ & $5 / a n$ & $=$ & $=$ & nd & $\% \mathrm{P}_{\text {ass }}=$ après 3 mois & \\
\hline 12 & Tropical sec, sabl. & Mulch de rameaux & 1 ou 2 (1 fois) & $(+)$ & $(+)$ & nd & & \\
\hline \multirow[t]{2}{*}{13} & $\begin{array}{l}\text { Tropical humide, } \\
\text { sablo-argileux }\end{array}$ & Mulch de copeaux & Non indiqué & + & nd & nd & $\begin{array}{l}\text { Humidité }=\text {, densité -, } \\
\text { érosion -, } \\
\% \text { Pass = à } 1 \text { an }\end{array}$ & $\% \mathrm{C}+$ à 1 an \\
\hline & & & & & & & $\mathrm{CEC}+, \%$ nitrate+, $\mathrm{pH}=$ & \\
\hline 14 & $\begin{array}{l}\text { Tropical humide, } \\
\text { sablo-argileux }\end{array}$ & Mulch de copeaux & $50 / a n$ & $(+)$ & + & nd & $\begin{array}{l}\text { Humidité }+ \text {, densité }= \\
\text { et infiltration }+ \text { an } 1 \text {; } \\
\text { CEC }+ \text {, stock } P_{\text {ass }}(-) \text { et } \mathrm{pH}+\text { an2 }\end{array}$ & Stocks $\mathrm{C}+$ et $\mathrm{N}(+)$ à 2 ans \\
\hline 15 & $\begin{array}{l}\text { Tropical humide, } \\
\text { limono-argileux }\end{array}$ & $\begin{array}{l}\text { Mulch de copeaux } \\
\text { et fumier sans NK }\end{array}$ & 49 an1, 19 an2 & + & + & nd & $\begin{array}{l}\mathrm{pH}=, \% \mathrm{~K}_{\text {éch }}=, \% \mathrm{Ca}_{\text {éch }}+ \\
\text { et } \% \mathrm{Mgéch}^{+} \text {an1 et an2 }\end{array}$ & $\% \mathrm{C}$ et $\mathrm{N}$ an1(+), an2+ \\
\hline
\end{tabular}

Les symboles + et - signalent des effets significatifs $(p<0,05),(+)$ et $(-)$ des effets non significatifs, $=l^{\prime}$ absence d'effet.

nd non déterminé ; * quand la source citée n'exprime pas l'apport en tMS, I'humidité a été estimée à $50 \%$; ** estimé d'après le volume; ${ }^{1}$ rendement de la première, deuxième ou quatrième

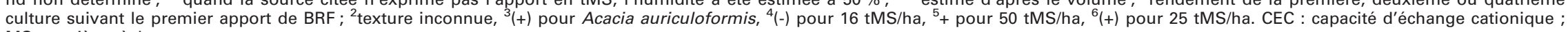
MS : matière sèche.

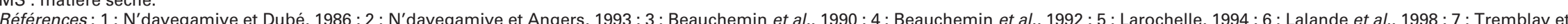

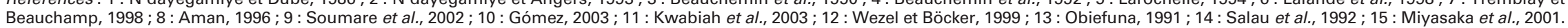




\section{Mulch en zone tropicale}

Sur sol sableux du Niger (PAM : 500 mm) en culture continue de mil, Wezel et Böcker (1999) montrent qu'un mulch de 1 et 2 tMS/ha de branches de Guiera senegalensis permet une augmentation de $70-80 \%$ (non significative) du rendement des deux cultures suivantes.

Sur sol limono-argileux du Vietnam (PAM : $1800 \mathrm{~mm}$ ), Hoang Fagerström et al. (2001) mesurent un rendement en riz pluvial plus élevé avec mulch de BRF de Tephrosia candida (légumineuse) qu'en couloir avec Tephrosia ou qu'après jachère améliorée à Tephrosia.

Les autres travaux recensés concernent des mulchs de copeaux de bois d'origine probablement non raméale. Sur sol sabloargileux du Nigeria (PAM : $1800 \mathrm{~mm}$ ) cultivé en ananas avec apport d'engrais NPK, Obiefuna (1991) note que le rendement est significativement plus élevé avec copeaux qu'avec balle de riz (+ 12 à $43 \%)$ ou sans mulch (+ 64 à $87 \%)$.

Sur sol sablo-argileux du Nigeria (PAM : $2500 \mathrm{~mm}$ ) sous bananier avec apport d'engrais NK, Salau et al. (1992) montrent qu'un mulch de $50 \mathrm{tMS} / \mathrm{ha}$ de copeaux permet d'augmenter les récoltes successives de 26 et $40 \%$ (significatif la seconde année) par rapport au témoin sans mulch ; avec mulch de paille l'augmentation est plus forte (65-82\%) et significative dès la première récolte.

Sur Andosol limono-argileux d'Hawaii (PAM : $2500 \mathrm{~mm}$ ) après canne à sucre, Miyasaka et al. (2001) observent que le rendement d'une culture de taro fertilisée en $\mathrm{P}$ est significativement plus élevé avec mulch de copeaux (50 et $20 \mathrm{tMS} / \mathrm{ha}$ en années 1 et 2 ) ou d'ensilage (20 puis $28 \mathrm{tMS} / \mathrm{ha}$ ) contenant un peu de fumier $(<2 \%)$ qu'avec fertilisation minérale (apport de $\mathrm{N}$ équivalent), surtout la seconde année (+ $100 \%)$; mais cette augmentation ne compense pas celle des coûts de production. Plusieurs types de pourritures ont un développement significativement plus important avec mulch qu'avec engrais, en relation probable avec l'humidité accrue du sol.

\section{Résumé des effets sur la plante cultivée}

La figure 1 présente une synthèse sur les variations de rendement en fonction de l'apport de BRF. L'enfouissement de BRF dans les sols à texture légère, surtout testé en milieu tempéré, provoque une baisse du rendement de la première culture.
Cette baisse est expliquée par une immobilisation de l'azote par la microflore, qui peut être compensée par des apports de N minéral ou organique. En revanche, le rendement des cultures suivantes est souvent supérieur à celui du témoin. L'enfouissement de BRF dans des sols moins sableux ou leur apport en mulch (même sur sol sableux) ont un effet positif sur le rendement dès la première culture ; ce résultat, obtenu en conditions tropicales, doit être confirmé en climat tempéré. L'intérêt de BRF riches en N (légumineuses) n'est pas avéré.

\section{Effets sur les propriétés physiques et hydriques du sol}

\section{Enfouissement en zone tempérée (Canada)}

Sur sol sablo-limoneux, Lalande et al. (1998) relèvent une augmentation significative de l'humidité dans l'horizon 0-15 cm les deux années suivant l'apport

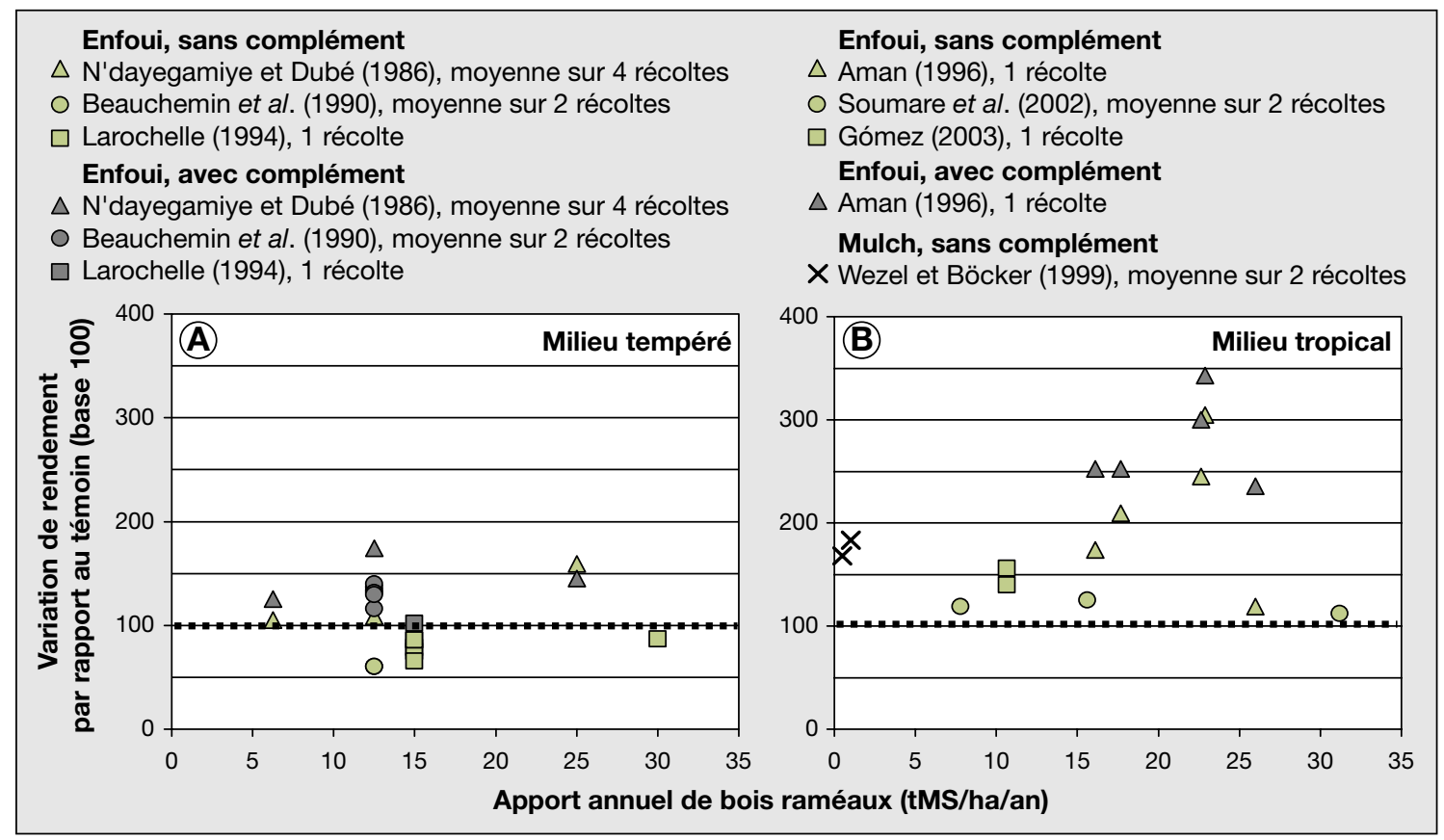

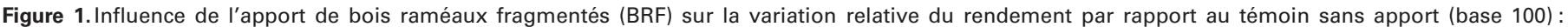
A) tempéré ; B) tropical.

Figure 1. Effect of soil amendment with small branches on the relative variation of mean yield as compared to control treatment without any input (basis 100): A) temperate; B) tropical. 
de BRF. La seconde année après enfouissement de BRF en sol sableux, Beauchemin et al. (1990) et Tremblay et Beauchamp (1998) notent aussi une humidité dans l'horizon 0-20 cm significativement plus élevée que sans apport organique ; les seconds ont aussi mesuré l'humidité la première année, sans observer de différence entre traitements.

Lalande et al. (1998) observent également une augmentation significative du taux de macroagrégats $(>0,25 \mathrm{~mm})$ stables dans l'horizon $0-15 \mathrm{~cm}$ la seconde année suivant l'apport de BRF. Mais après neuf années d'apports biennaux de BRF avec ou sans apport azoté sur sol sableux cultivé en céréales, N'dayegamiye et Angers (1993) n'observent aucun effet significatif sur la macroagrégation dans l'horizon 0-15 cm; ils suggèrent que celle-ci est peu affectée par l'humine stable produite par décomposition des BRF.

\section{Enfouissement en zone tropicale}

À la deuxième récolte suivant les apports dans un sol sableux du Sénégal (PAM : $700 \mathrm{~mm}$ ), Soumare et al. (2002) notent l'augmentation significative de la capacité de rétention en eau avec BRF par rapport aux témoins avec engrais ou sans apport (+ 21 à $24 \%$ ) et la diminution significative de la densité apparente par rapport au témoin sans apport (- 8 à - 13\%).

\section{Mulch en zone tropicale}

Sur sol sablo-argileux du Nigeria cultivé en ananas (PAM : $1800 \mathrm{~mm}$ ), Obiefuna (1991) note qu'un an après les apports, la densité apparente dans l'horizon 0-20 cm est significativement moins élevée avec copeaux qu'avec balle de riz (- $6 \%)$ ou sans mulch (-8\%). Les mulchs affectent peu la capacité de rétention bien qu'ils augmentent l'humidité à la capacité au champ et au point de flétrissement. En revanche, l'érosion est significativement moindre avec copeaux qu'avec balle de riz (- $53 \%$ ) ou sans mulch (- $69 \%)$.

Sur une situation voisine sous bananier (PAM : 2500 mm), Salau et al. (1992) constatent que l'infiltration est significativement plus importante avec copeaux qu'avec paille ou sans mulch, les différences diminuant au fil des mois. Sur l'année, l'humidité du sol est plus forte avec paille qu'avec copeaux (effet attribué à une meilleure couverture du sol), et avec copeaux que sans mulch. En revanche la densité apparente dans l'horizon 0-10 cm ne diffère pas entre traitements. De plus, la température du sol à $5 \mathrm{~cm}$ de profondeur varie moins sous copeaux que sous paille, et sous paille que sans mulch.

Sur sol sablo-argileux encroûté du Burkina Faso portant une savane herbacée (PAM : 500 mm), Mando (1997) note, deux ans après l'installation d'un mulch, que la porosité dans l'horizon 0-5 cm est peu affectée par sa présence ou sa nature (herbacée et/ou ligneuse). Mais, en général, la conductivité hydraulique et l'humidité du sol diminuent et la compacité augmente du mulch ligno-herbacé au mulch herbacé puis au mulch ligneux, surtout en présence de termites; en l'absence de termites, les différences entre traitements sont souvent faibles.

\section{Résumé des effets sur les propriétés physiques et hydriques du sol}

L'apport de BRF améliore généralement les propriétés physiques et hydriques du sol par rapport aux témoins sans apport organique : infiltration, humidité, porosité et stabilité structurale plus élevées, compacité plus faible, température plus stable; en mulch, les apports ligneux contribuent aussi à diminuer l'érosion. Mais l'intérêt des mulchs ligneux par rapport aux mulchs herbacés n'est pas net, plutôt favorable en termes de porosité et d'érosion, mais incertain ou négatif en termes d'infiltration et d'humidité du sol. Dans les zones tropicales sèches, l'effet des apports est très influencé par la présence des termites.

\section{Effets sur les propriétés chimiques et d'échange du sol}

\section{Enfouissement en zone tempérée (Canada)}

Sur sol sableux cultivé en pomme de terre recevant $\mathrm{P}$ et $\mathrm{K}$ minéraux, Beauchemin et al. (1992a) notent la moindre minéralisation de $\mathrm{N}$ organique du sol et l'utilisation accrue de $\mathrm{N}$ de l'engrais par la culture avec
BRF ; l'immobilisation de $\mathrm{N}$ minéral est plus élevée avec BRF frais que composté. Sur sol limono-argileux cultivé en orge, Larochelle (1994) constate la diminution de la teneur en nitrate $\left(\mathrm{NO}_{3}{ }^{-}\right)$dans l'horizon $0-15 \mathrm{~cm}$ avec apport de BRF sans complément azoté.

Sur sol sablo-limoneux cultivé en oignon puis blé avec fertilisation complète, Lalande et al. (1998) observent une teneur en $\mathrm{NO}_{3}{ }^{-}$dans l'horizon $0-15 \mathrm{~cm}$ significativement inférieure après enfouissement de 150 tMS/ha de BRF (feuillus); cet effet disparaît la seconde année. La corrélation négative (significative) entre teneur en $\mathrm{NO}_{3}{ }^{-}$et nombre de colonies microbiennes témoigne de leur rôle dans l'immobilisation de $\mathrm{N}$.

Les travaux de N'dayegamiye et Dubé (1986) sur sol sablo-graveleux cultivé en blé puis prairie et ceux de Beauchemin et al. (1990) sur sol sableux cultivé en pomme de terre montrent que l'immobilisation de $\mathrm{N}$ est moins nette durant la seconde culture suivant l'apport de BRF, comme l'atteste l'augmentation des rendements et des prélèvements de $\mathrm{N}$ par les cultures. Par ailleurs, après quatre années de culture comportant deux apports de BRF, N'dayegamiye et Dubé (1986) ne constatent pas d'effet significatif sur la capacité d'échange cationique (CEC) du sol.

Sur sol sableux cultivé en pomme de terre avec fertilisation complète après prairie, Tremblay et Beauchamp (1998) notent que l'apport de 37,5 tMS/ha de BRF s'accompagne d'une diminution significative de teneur en $\mathrm{P}$ assimilable $\left(\mathrm{P}_{\mathrm{ass}}\right)$ dans l'horizon 0-20 cm, qu'ils attribuent à son immobilisation par la microflore. Cette diminution perdure l'année suivante, avec ou sans second apport, mais n'est plus significative. Par ailleurs, le $\mathrm{pH}$ du sol n'est pas modifié.

\section{Enfouissement en zone tropicale}

Après deux cultures de tomate suivant une application de BRF dans un sol sableux du Sénégal (PAM : 700 mm), Soumare et al. (2002) observent l'augmentation de la CEC et de la teneur en K échangeable $\left(\mathrm{K}_{\text {éch }}\right)$ et la diminution de la teneur en $\mathrm{P}_{\text {ass }}$, toutes significatives. Les données sur les rendements et les teneurs en $\mathrm{N}$ et $\mathrm{P}$ des feuilles suggèrent une immobilisation de $\mathrm{N}$ et $\mathrm{P}$ par la microflore du sol pendant la première culture, puis une minéralisation les rendant disponibles pour la seconde culture. 
Ces résultats sont contredits par ceux de Kwabiah et al. (2003) sur sol argilosableux du Kenya (PAM : $1800 \mathrm{~mm}$ ) cultivé en maïs, dans lequel la teneur en $\mathrm{P}_{\text {ass }}$ est corrélée positivement à la quantité de $\mathrm{P}$ apporté par les BRF. Quelques mois après l'apport ligneux, le sol contient autant de $\mathrm{P}_{\text {ass }}$ qu'après apport minéral de $25 \mathrm{kgP} / \mathrm{ha}$.

\section{Mulch en zone tropicale}

Les travaux mentionnés concernent des mulchs de copeaux d'origine probablement non raméale. Sur sol sablo-argileux du Nigeria (PAM : $1800 \mathrm{~mm}$ ) portant une culture d'ananas fertilisée, Obiefuna (1991) observe dans l'horizon 0-20 cm une CEC similaire après mulch de copeaux ou de balle de riz, significativement plus élevée que sans mulch. La teneur en $\mathrm{NO}_{3}{ }^{-}$est significativement supérieure avec mulch (mais la densité apparente est plus faible, et les différences de stock ne semblent pas significatives). La teneur en $\mathrm{P}_{\text {ass }}$ est significativement plus élevée avec balle de riz qu'avec copeaux, les autres différences n'étant pas significatives.

Sur une situation voisine (PAM : $2500 \mathrm{~mm}$ ), deux ans après plantation de bananier fertilisé en $\mathrm{N}$ et $\mathrm{P}$, Salau et al. (1992) observent que, dans l'horizon 0-15 cm, la CEC est similaire après mulch de copeaux ou de paille, et significativement plus élevée que sans mulch; le $\mathrm{pH}$ est significativement supérieur et le stock de $\mathrm{P}_{\text {ass }}$ tend à être moindre avec copeaux qu'avec paille ou sans mulch.

Sur Andosol limono-argileux d'Hawaii cultivé en taro avec apport de P (PAM : 2500 mm), Miyasaka et al. (2001) n'observent pas d'effet des traitements (mulch de copeaux ou d'ensilage, ou engrais) sur le $\mathrm{pH}$ et la teneur en $\mathrm{K}_{\text {éch }}$ lors des deux premières récoltes, mais les teneurs en calcium et magnésium échangeables sont significativement supérieures avec mulch (+ 100 \%).

\section{Résumé des effets sur les propriétés chimiques et d'échange du sol}

L'enfouissement de BRF en zone tempérée, testé sur des sols à texture légère, conduit à l'immobilisation de $\mathrm{N}$ et $\mathrm{P}$, surtout l'année de l'apport. Mais pH et CEC ne semblent pas affectés. En zone tropi- cale, les apports ligneux ou herbacés ont peu d'effet sur $\mathrm{NO}_{3}{ }^{-}$et $\mathrm{P}_{\text {ass }}$, surtout lorsque les stocks (kg/ha) plutôt que les teneurs ( $\mathrm{g} / \mathrm{kg}$ ) sont considérés. En revanche, pH et CEC tendent à être plus élevés après apport ligneux que sans apport organique ou même qu'après apport herbacé.

\section{Effets sur la matière organique du sol}

\section{Enfouissement en zone tempérée (Canada)}

Après quatre ans de culture de céréales ou prairie et deux apports de BRF sur sol sablo-graveleux fertilisé en $\mathrm{P}$ et $\mathrm{K}$, N'dayegamiye et Dubé (1986) constatent en général avec BRF des augmentations significatives de la teneur en $\mathrm{C}$ total $\left(\mathrm{C}_{\mathrm{t}} ;+20\right.$ à $\left.70 \%\right)$ et du rapport $\mathrm{C} / \mathrm{N}$ du sol (+ 12 à $24 \%)$, tandis que la teneur en $\mathrm{N}$ total $\left(\mathrm{N}_{\mathrm{t}}\right)$ ne varie pas significativement (+ 8 à $40 \%$ ). Ils notent que l'apport conjoint de lisier favorise la décomposition et l'humification des BRF.

Sur sol sablo-limoneux cultivé en oignon puis blé fertilisé, Lalande et al. (1998) observent avec BRF des augmentations significatives des teneurs en $\mathrm{C}_{\mathrm{t}}(+17 \%)$ et $\mathrm{N}_{\mathrm{t}}(+11 \%)$ dans l'horizon $0-15 \mathrm{~cm} \mathrm{la}$ deuxième année, mais pas la première.

Sur sol sableux cultivé en pomme de terre avec fertilisation complète, Tremblay et Beauchamp (1998) constatent que la teneur en $\mathrm{C}_{t}$ dans l'horizon 0-20 cm n'est pas affectée par l'apport de BRF la première année; l'année suivante, avec ou sans second apport, elle est significativement supérieure avec BRF (+ $10 \%$ en moyenne); le rapport $\mathrm{C} / \mathrm{N}$ n'est pas affecté.

Après neuf années d'apports biennaux de BRF avec ou sans apports complémentaires de $\mathrm{N}$ sur sol sableux cultivé en céréales, N'dayegamiye et Angers (1993) observent que la teneur en $\mathrm{C}_{\mathrm{t}}$ dans l'horizon $0-15 \mathrm{~cm}$ est significativement supérieure avec BRF (+ 16 à 37 \%). Sans apport azoté, la teneur en $\mathrm{N}_{\mathrm{t}}$ est significativement supérieure avec BRF (+ 9 à $27 \%$ ), mais pas le rapport $\mathrm{C} / \mathrm{N}(0$ à $+10 \%)$; avec apport azoté, le rapport $\mathrm{C} / \mathrm{N}$ est significativement supérieur avec BRF (+ 6 à $18 \%$ ) mais pas la teneur en $\mathrm{N}_{\mathrm{t}}(-8$ à $+25 \%)$. Avec BRF, les quantités respectives de $\mathrm{C}$ dans les fractions lourdes et légères aug- mentent de 17 et $38 \%$ et la teneur en humine de $38 \%$, mais la teneur en acides humiques n'est pas affectée ; 80 \% des différences entre traitements sont imputables aux fractions lourdes et humine.

\section{Enfouissement en zone tropicale}

Dans un sol sableux du Sénégal (PAM : $700 \mathrm{~mm}$ ), lors de la deuxième récolte suivant l'enfouissement d'apports organiques, Soumare et al. (2002) notent que la teneur en $C_{t}$ avec BRF est significativement plus faible qu'avec apport annuel d'engrais ternaire ou de litière compostée (- $40 \%)$, mais ne diffère pas statistiquement de celle mesurée sans apport (quoique $40 \%$ supérieure). En revanche, la teneur en $\mathrm{N}_{t}$ avec BRF est significativement plus élevée en général qu'avec litière compostée $(+100 \%)$ ou sans apport $(+1000 \%)$, mais ne diffère pas de celle mesurée après apport annuel d'engrais (quoique 20\% supérieure). L'effet des BRF est donc plus marqué ici sur N que sur C. Les différences de stocks de $C_{t}$ et $N_{t}$ entre traitements semblent équivalentes aux différences de teneurs.

\section{Mulch en zone tropicale}

Les travaux mentionnés concernent des mulchs de copeaux d'origine probablement non raméale. Plus d'un an après des apports en mulch sur sol sabloargileux du Nigeria cultivé en ananas fertilisé (PAM : 1800 mm), Obiefuna (1991) relève que la teneur en $C_{t}$ dans l'horizon 0-20 cm est significativement plus élevée avec balle de riz que copeaux $(+11 \%)$ et avec copeaux que sans mulch $(+5 \%)$; toutefois, la densité apparente étant plus faible avec copeaux, les stocks de $C_{t}$ avec copeaux ou sans mulch sont peu différents $( \pm 2 \%)$.

Dans une situation voisine sous bananier (PAM : $2500 \mathrm{~mm}$ ), Salau et al. (1992) constatent que le stock de $C_{t}$ dans l'horizon $0-15 \mathrm{~cm}$ est significativement plus élevé avec copeaux (+ $30 \%)$ ou paille (+ $40 \%)$ que sans mulch deux ans après la plantation, mais les différences entre traitements ne sont pas significatives avant cette date ; sur la période, les différences de stocks de $\mathrm{N}_{t}$ ne sont jamais significatives. Les traitements n'ont pas d'effet significatif sur la densité apparente et affectent donc similairement teneurs et stocks. 
Sur Andosol limono-argileux d'Hawaii cultivé en taro avec engrais phosphaté (PAM : 2500 mm), Miyasaka et al. (2001) observent, lors de la récolte qui suit le deuxième apport, que les teneurs en $\mathrm{C}$ organique et $\mathrm{N}_{t}$ sont significativement plus importantes avec mulch de copeaux ou d'ensilage qu'avec apport de $\mathrm{N}$ minéral (respectivement +17 et $+8 \%$ pour $\mathrm{C}$, significativement plus élevé avec copeaux qu'avec ensilage $;+8$ et $+4 \%$ pour $\mathrm{N}$ ) ; mais il n'y a pas de différence significative entre traitements lors de la récolte qui suit le premier apport.

\section{Résumé des effets sur la matière organique du sol}

La figure 2 présente une synthèse sur les variations de teneur en $\mathrm{C}$ du sol en fonction de l'apport de BRF. Par rapport aux témoins sans apport organique ou sans aucun apport, les BRF déterminent une augmentation de la teneur en $\mathrm{C}$ du sol, faible l'année de l'apport (0 à $10 \%$ en général), le plus souvent significative l'année suivante (10 à $40 \%$ ) et au-delà (20 à $70 \%$ avec apports renouvelés). L'augmentation de la teneur en $\mathrm{N}_{\mathrm{t}}$ du sol est imper- ceptible l'année de l'apport, faible la deuxième année (0 à $10 \%$ en général), parfois significative au-delà (10 à $40 \%$ avec apports renouvelés). Les variations de stocks de $\mathrm{C}$ et $\mathrm{N}$ sont moins nettes, du fait d'une densité apparente souvent inférieure avec BRF. L'augmentation du rapport $\mathrm{C} / \mathrm{N}$ ne devient significative qu'à partir de la quatrième année (10 à $20 \%$ avec apports renouvelés). L'humification des BRF est favorisée par des apports azotés simultanés. L'intérêt des BRF par rapport aux apports herbacés ou azotés n'est pas avéré.

\section{Effets sur l'activité biologique du sol}

\section{Enfouissement en zone tempérée (Canada)}

Dans la couche 0-20 cm d'un sol sableux sous pomme de terre fertilisée, Tremblay et Beauchamp (1998) notent que 6 et 12 mois après apport de BRF la population de champignons a respectivement triplé et doublé par rapport au témoin, mais n'est plus affectée significativement ensuite (avec ou sans second apport). Les populations de bactéries et d'actinomycètes ne sont pas affectées significativement. La biomasse microbienne est plus élevée avec BRF, mais la différence n'est significative qu'à certaines périodes. Dans la couche $0-15 \mathrm{~cm}$ d'un sol sablolimoneux cultivé en oignon puis blé fertilisé, Lalande et al. (1998) observent que les populations de bactéries, actinomycètes et surtout champignons sont significativement plus importantes avec BRF l'année de l'apport (+ 140, 163 et 2300 \%, respectivement) ; l'année suivante, la différence est significative pour les champignons seulement (+ 48, 21 et $700 \%$, respectivement). De fortes variations sont observées entre dates, avec des maxima 2 mois après apport. L'activité phosphatase alcaline n'est pas affectée l'année de l'apport, mais, l'année suivante, elle est significativement plus élevée avec BRF. Dans la couche $0-5 \mathrm{~cm}$ d'un sol limonoargileux cultivé en orge, Larochelle (1994) observe que la mésofaune est plus abondante avec BRF (jusqu'à 23 fois), en particulier les acariens et collemboles (fongivores et leurs prédateurs), avec des variations sensibles selon les essences utilisées, le diamètre des rameaux, la taille des copeaux, l'apport éventuel de N, etc.

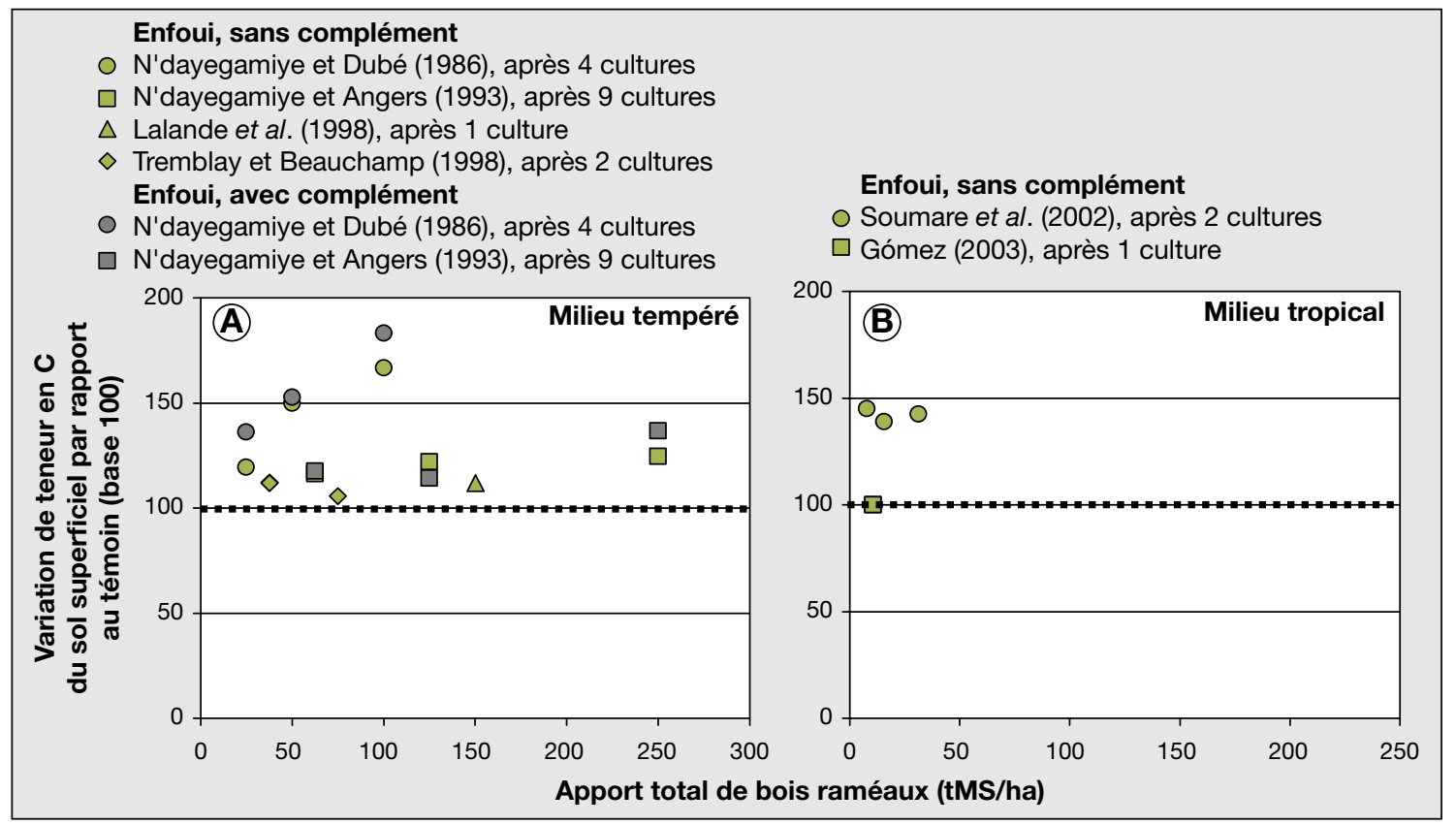

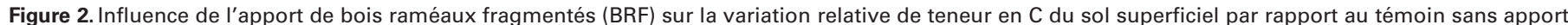
(base 100) : A) tempéré ; B) tropical.

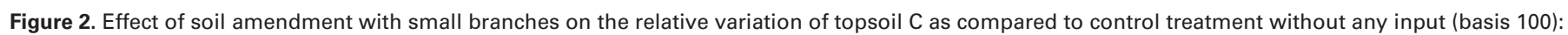
A) temperate; B) tropical. 


\section{Enfouissement ou mulch en zone tropicale}

Les travaux de Mando (1997) au Burkina Faso soulignent l'importance des termites dans l'évolution du sol après mulch organique.

\section{Résumé des effets sur l'activité biologique du sol}

Les travaux réalisés au Canada montrent que l'enfouissement de BRF stimule le développement des populations de champignons, d'insectes qui leur sont liés, et, à un moindre niveau, de bactéries et d'actinomycètes, surtout l'année de l'apport. Ce sujet a été peu étudié en zone tropicale.

\section{Conclusion}

L'apport de BRF enrichit le sol en matière organique et en nutriments, ce qui stimule l'activité biologique, notamment fongique ; cette stimulation améliore ensuite la disponibilité des nutriments pour les plantes. Mais les BRF peuvent provoquer une immobilisation de $\mathrm{N}$, voire de $\mathrm{P}$, l'année du premier apport, donc une baisse du rendement, observée surtout en cas d'enfouissement en sol sableux sous climat tempéré ; cette immobilisation peut être limitée en cas d'apport azoté simultané. Cependant, le rendement est très généralement accru l'année suivante, même en cas de nouvel apport ligneux. Par ailleurs, surtout en mulch, les BRF ont un effet favorable sur les propriétés physiques et hydriques du sol.

De nombreux facteurs modulent ces effets, dans des proportions mal connues : espèces utilisées, diamètre des rameaux, dimension des fragments, dose, etc. Il semble néanmoins que l'utilisation de légumineuses n'ait pas d'effet particulièrement favorable.

Enfin, les BRF n'ont pas d'intérêt clairement décisif par rapport aux apports herbacés (propriétés physico-hydriques ou organiques du sol) ou azotés (statut organique du sol, rendement).

\section{Remerciements}

Les auteurs remercient Gilles Lemieux et Benoît Noël pour la mise à disposition de leurs fonds documentaires. Ce travail a été soutenu financièrement par l'Institut de recherche pour le développement (IRD) et par l'Institut des sciences et industries du vivant et de l'environnement (AgroParisTech).

\section{Références}

Altieri MA. Agroecology: the science of natural resource management for poor farmers in marginal environments. Agr Ecosyst Environ 2002 ; 93: 1-24.

Aman SA. Effects of chopped twig wood on maize growth and yields in the forest-savanna transition zone of Côte d'Ivoire. Québec : université Laval, Groupe de coordination sur les bois raméaux, 1996. www.hydrogeochem.qc.ca/ pages/publications_gcbr/doc169.pdf

Beauchemin S, N'dayegamiye A, Laverdière MR Effets $d^{\prime}$ apport $d$ 'amendements ligneux frais et humifiés sur la production de pomme de terre et sur la disponibilité de l'azote en sol sableux. Can J Soil Sci 1990 ; 70 : 555-64.

Beauchemin S, N'dayegamiye $A$, Laverdière MR. Effets d'amendements ligneux sur la disponibilité d'azote dans un sol sableux cultivé en pommes de terre. Can J Soil Sci 1992a ; 72 : 89-95.

Beauchemin S, N'dayegamiye A, Laverdière MR. Phytotoxicité des matériaux ligneux frais et compostés utilisés comme amendements organiques des sols. Can J Soil Sci 1992b ; 72 : 177-81.

Ewel JJ. Natural systems as models for the design of sustainable systems of land use. Agroforest Syst $1999 ; 45: 1-21$.

Gómez CER. Comparison of two sources of ramial chipped wood on maize (Zea mays) yield. Québec: université Laval, Groupe de coordination sur les bois raméaux, 2003. www.hydrogeochem.qc.ca/pages/publications_gcbr/doc170.pdf

Höang Fagerström $\mathrm{MH}$, van Noordwijk $\mathrm{M}$, Phien $T$, Vinh NC. Innovations within upland rice-based systems in northern Vietnam with Tephrosia candida as fallow species, hedgerow, or mulch: net returns and farmers' response. Agr Ecosyst Environ $2001 ; 86$ : 23-37.

Izac AMN, Swift MJ. On agricultural sustainability and its measurement in small-scale farming in sub-Saharan Africa. Ecol Econ 1994; 11: 105-25.

Kumar K, Goh KM. Crop residues and management practices: Effects on soil quality, soil nitrogen dynamics, crop yield, and nitrogen recovery. Adv Agron 2000 ; 68 : 197-319.

Kwabiah AB, Stoskopf NC, Palm CA, Voroney RP, Rao MR, Gacheru E. Phosphorus availability and maize response to organic and inorganic fertilizer inputs in a short term study in western Kenya. Agr Ecosyst Environ 2003 ; 95 : 49-59.
Lalande R, Furlan V, Angers DA, Lemieux G. Soil improvement following addition of chipped wood from twigs. Am J Alternative Agr 1998; $13: 132-7$.

Larochelle L. L'impact du bois raméal fragmenté sur la dynamique de la mésofaune du sol. Québec: université Laval, faculté des études supérieures, Rapport de maître ès sciences (M.Sc.), 1994. www.hydrogeochem.qc.ca/pages/ publications_gcbr/doc78.pdf

Lemieux G. Cet univers caché qui nous nourrit: le sol vivant. Québec: université Laval, Groupe de coordination sur les bois raméaux, 1996. http://www.sbf.ulaval.ca/brf/cet_univers.html

Mando A. Effect of termites and mulch on the physical rehabilitation of structurally crusted soils in the Sahel. Land Degrad Dev 1997; 8: 269-78.

Manlay RJ, Feller C, Swift MJ. Historical evolution of soil organic matter concepts and their relationships with the fertility and sustainability of cropping systems. Agr Ecosyst Environ 2007; $119: 217-33$.

Miyasaka SC, Hollyer JR, Kodani LS. Mulch and compost effects on yield and corm rots of taro. Field Crop Res 2001 ; 71 : 101-12.

N'dayegamiye A, Dubé A. L'effet de l'incorporation de matières ligneuses sur l'évolution des propriétés chimiques du sol et sur la croissance des plantes. Can J Soil Sci 1986; 66 : 623-31.

N'dayegamiye A, Angers DA. Organic matter characteristics and water-stable aggregation of a sandy loam soil after 9 years of woodyresidue applications. Can J Soil Sci 1993; 73: 115-22.

Obiefuna JC. Establishment of pineapple orchards and soil loss control systems for erodible tropical ultisols of southeastern Nigeria. Fruits $1991 ; 46: 145-51$.

Odum EP. The strategy of ecosystem development. Science 1969 ; 164 : 262-70.

Salau OA, Opara-Nadi OA, Swennen R. Effects of mulching on soil properties, growth and yield of plantain on a tropical ultisol in southeastern Nigeria. Soil Till Res $1992 ; 23$ : 73-93.

Soumare MD, Mnkeni PNS, Khouma M. Effects of Casuarina equisetifolia composted litter and ramial-wood chips on tomato growth and soil properties in Niayes, Senegal. Biol Agric Hortic $2002 ; 20: 111-23$.

Tilman D, Cassman KG, Matson PA, Naylor R, Polasky S. Agricultural sustainability and intensive production practices. Nature $2002 ; 418$ : 671-7.

Tremblay J, Beauchamp CJ. Fractionnement de la fertilisation azotée d'appoint à la suite de l'incorporation au sol de bois raméaux fragmentés: modifications de certaines propriétés biologiques et chimiques d'un sol cultivé en pomme de terre. Can J Soil Sci 1998 ; 78 : 275-82.

Wezel A, Böcker R. Mulching with branches of an indigenous shrub (Guiera senegalensis) and yield of millet in semi-arid Niger. Soil Till Res $1999 ; 50: 341-4$. 